\title{
Artrodesis de cuatro esquinas de la muñeca
}

\author{
P. J. Delgado, F. A. Figueredo, A. Díez-Montiel, M. Gil de Rozas, J. R. Truan \\ Unidad de Cirugía de la Mano y del Miembro Superior. Servicio de Cirugía Ortopédica y Traumatología \\ Hospital Universitario Madrid Montepríncipe. Universidad San Pablo CEU. Madrid. España.
}

Resumen: La artrosis de la muñeca es una de las patologías más frecuentes y precisa un estudio detallado de la misma para realizar el tratamiento más adecuado. La artrodesis de cuatro esquinas con resección del escafoides es un procedimiento aceptable para tratar los cambios degenerativos, especialmente en la muñeca SLAC/SNAC. El objetivo es fusionar las articulaciones mediocarpianas dolorosas manteniendo la movilidad a través de la articulación radiolunar. Las técnicas quirúrgicas se han ido desarrollando a lo largo de las últimas décadas y los resultados funcionales han mejorado, pero no sus complicaciones. En este artículo se presentan los métodos de fijación y se revisa su evidencia científica en la literatura, así como los nuevos conceptos y técnicas, como la artroscopia.

Palabras clave: Muñeca, artrodesis, cuatro esquinas, SNAC/SLAC.

\section{Four-corner fusion of the wrist}

\begin{abstract}
Osteoarthritis of the wrist is one of the most common conditions and requires careful analysis and specific indications and rationale for appropriate treatment. Four-corner arthrodesis with scaphoid excision has been shown to be an acceptable method for treating this degenerative changes, especially SLAC/SNAC wrist. The goal is to fuse the painful, diseased midcarpal joints and to preserve motion through the radiolunate joint. Surgical techniques have advanced over the last few decades, and the functional outcomes have improved but complications not decreased. In this article we discuss the fixation methods available and review the corresponding evidence in the literature. New implants and new concepts are also discussed, as arthroscopic-assisted technique.
\end{abstract}

Key words: Wrist, fusion, four-corner, SNAC/SLAC.

La artrodesis de las cuatro esquinas (A4E) fue inicialmente descrita por Watson en 1984 para el tratamiento del colapso avanzado del carpo', quien lo definió como "procedimiento SLAC". Consiste en la fusión de los huesos grande, ganchoso, semilunar y piramidal que convierte la muñeca en una articulación simple tipo cóndilo carpiano. De esta forma se evita la disminución de altura del carpo y la pérdida de fuerza asociada, manteniendo la movilidad de la muñeca.

La resección del escafoides incrementa la movilidad y se utiliza como injerto autólogo para incrementar la consolidación ${ }^{2}$, aunque en la necrosis avascular del hueso grande, las lesiones osteocondrales traumáticas de la cabeza del hueso grande y la inestabilidad mediocarpiana no siempre es necesario la extirpación del escafoides carpiano ${ }^{3}$. Watson inicialmente sustituía el escafoides por una prótesis de silicona para evitar la desviación radial, gesto que actualmente está abandonado por las complicaciones que se han visto asociadas como luxaciones, fracturas del implante y las reacciones a cuerpo extraño.

La principal indicación es la muñeca SLAC/SNAC tipos II y III, así como otros procesos degenerativos medio- carpianos que mantengan íntegra la articulación radiosemilunar. Está contraindicada de forma absoluta en la traslación cubital del carpo, muñeca SLAC secundaria a una enfermedad de Kienböck y afectación de la articulación entre radio y semilunar ${ }^{4,5}$.

La clave del éxito depende de la obtención de una fusión solida entre los cuatro huesos que se artrodesan. Con la fusión del hueso grande, ganchoso, semilunar y piramidal se estabiliza la mediocarpiana y se elimina el dolor originado por la artrosis entre el hueso grande y semilunar. Para la fijación se pueden utilizar agujas de Kirschner, tornillos canulados, placas cuadrangulares, grapas de memoria, injertos de corticoesponjosa de cresta iliaca atornillados ${ }^{5}$ y placas dorsales circulares atornilladas, que permiten estabilizar los huesos cruentados y evitar la pérdida de altura del carpo (a) Figura l.
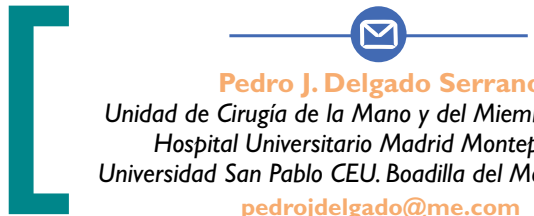

Unidad de Cirugía de la Mano y del Miembro Superior Hospital Universitario Madrid Montepríncipe Universidad San Pablo CEU. Boadilla del Monte, Madrid. pedrojidelgado@me.com 

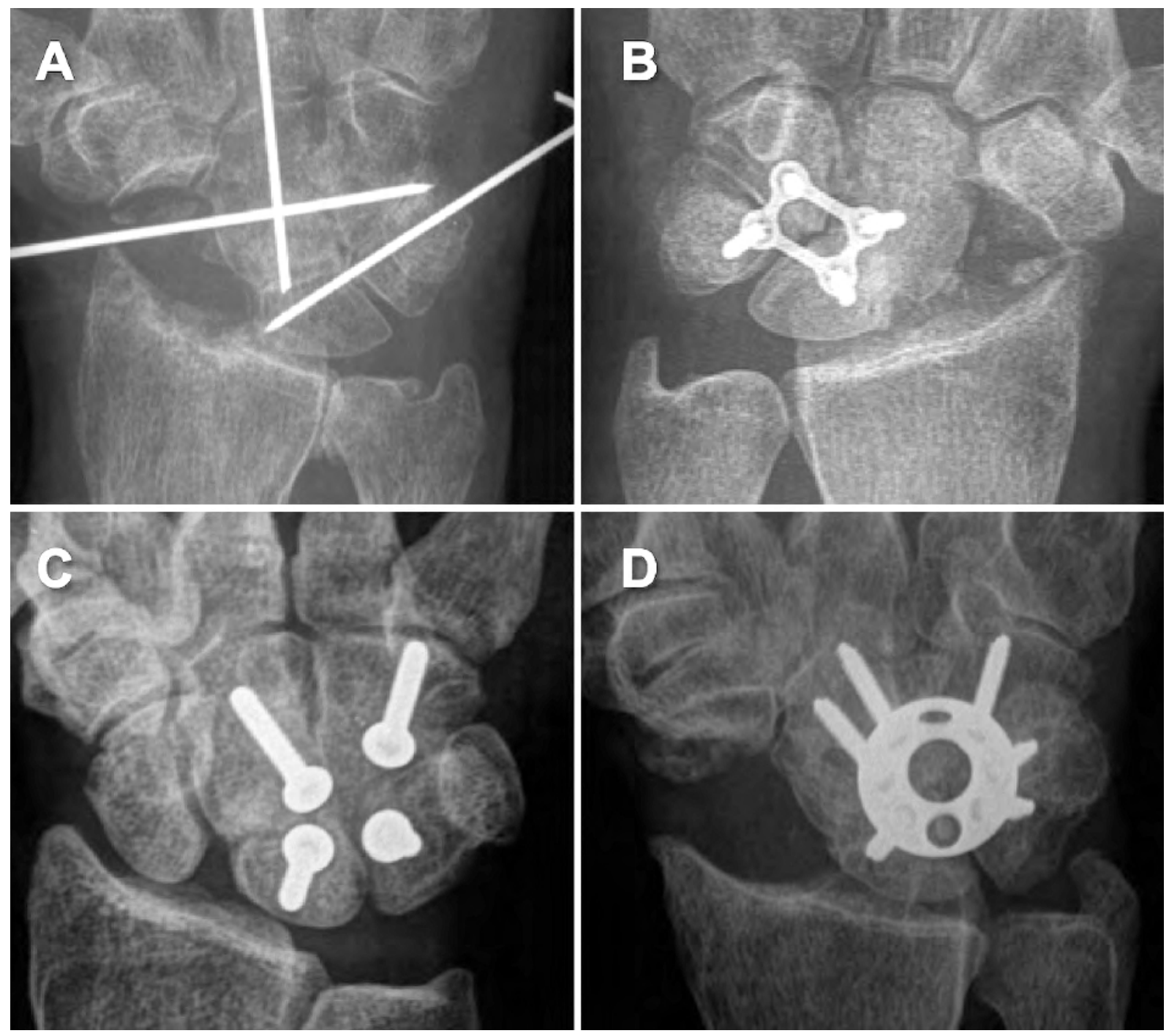

Figura. I. Imágenes radiológicas de diversas opciones de fijación de la artrodesis de cuatro esquinas: A. agujas de Kirschner, B. placa cuadrangular y tornillos de $2.3 \mathrm{~mm}$, C. injerto de cresta iliaca dorsal atornillado y D. placa dorsal circular atornillada.

Si bien no se trata de un concepto novedoso no ha dejado de ser un tema de actualidad. Es interesante ver el número de publicaciones aparecidas sobre esta técnica quirúrgica en los últimos veinte años, con momentos de gran entusiasmo con determinadas opciones de fijación, seguidas de períodos en el que el aumento de trabajos tenía como objetivo el desencanto respecto a la eficacia de dichos implantes. En los primeros años de la década del 2000 se retomó la técnica con la aparición de la placa circular Spider ${ }^{\circledR}$ (KMI, San Diego), con un repunte a finales de la década basado en la publicación de sus complicaciones y búsqueda de alternativas de fijación. En los últimos 5 años la A4E ha vuelto a la actualidad con la introducción de las técnicas artroscópicas y variantes de fusión mediocarpiana (2-esquinas, 3-esquinas o bicolumnar).

\section{BIOMECÁNICA Y NECESIDADES FUN- CIONALES}

La movilidad de la muñeca es la suma de dos articulaciones: radiocarpiana (la formada entre radio, cúbito y los huesos de la primera fila del carpo) y mediocarpiana (entre las dos hileras de huesos del carpo). En el eje sagital puro (flexo-extensión) en la articulación radiocarpianase origina el $62 \%$ de la flexión y el $50 \%$ de la extensión, mientras que en la mediocarpiana el $38 \%$ de la flexión y el 50\% de la extensión. Si bien la muñeca tiene un amplio rango de movilidad activo, la mayor parte de las actividades de la vida diaria se realizan con porcentaje menor que se encuentra aproximadamente los $70^{\circ}$ de flexo-extensión ${ }^{6-9}$ (2) Tabla I. 


\begin{tabular}{|c|c|c|c|c|}
\hline AUTOR (AÑO) & FLEXIÓN & EXTENSIÓN & DR & DC \\
\hline Brunfield y Champoux (1984) ${ }^{6}$ & $10^{\circ}$ & $35^{\circ}$ & $\mathrm{N} / \mathrm{D}$ & $\mathrm{N} / \mathrm{D}$ \\
\hline Palmer y cols $(1985)^{7}$ & $5^{\circ}$ & $30^{\circ}$ & $10^{\circ}$ & $15^{\circ}$ \\
\hline Nelson $(1990)^{8}$ & $28^{\circ}$ & $37^{\circ}$ & $12^{\circ}$ & $27^{\circ}$ \\
\hline Ryu y cols (1991) 9 & $40^{\circ}$ & $40^{\circ}$ & $10^{\circ}$ & $30^{\circ}$ \\
\hline
\end{tabular}

DR: desviación radial, DC: desviación cubital.

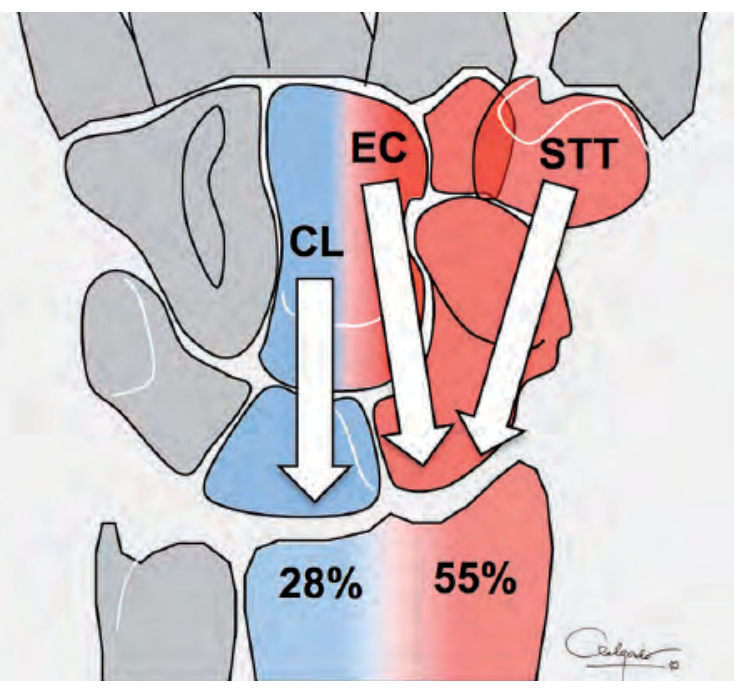

Figura 2. Transmisión de fuerzas en el eje axial de la muñeca. Nótese cómo el 55\% de las fuerzas se concentran en la fosa escafoidea del radio, procedentes de la articulación escafo-trapecio-trapezoide (STT) y de la escafo-capitate (EC). El $28 \%$ se localizan en la fosita semilunar del radio, procedentes de la articulación capitolunar (CL).

El eje verdadero funcional de la muñeca, que lleva la muñeca desde extensión-desviación radial a flexióndesviación cubital, ocurre en casi su totalidad a nivel de la articulación mediocarpiana. Este efecto justifica que artrosis radiocarpianas muy evolucionadas pueden ser indoloras, cuando la movilidad se traslada a nivel de la mediocarpiana ${ }^{10}$. Las articulaciones metacarpofalángicas del segundo y tercer radios son inmóviles y estables. Recogen la mayoría de las presiones generadas durante la acción de agarre que transmiten hacia la columna radial del carpo formada por trapecio, trapezoide y hueso grande $y$, a su vez, a escafoides $y$ semilunar @ Figura 2. Los radios cuarto y quinto son móviles, inestables y tienen como función aumentar la movilidad y la superficie de agarre. En la articulación radiocarpiana se localizan el $83 \%$ de las cargas axiales que actúan sobre la muñeca, de las que el $22 \%$ proceden de la articulación hueso grande-semilunar, el 33\% de la hueso grande-escafoides y el 33\% de la escafo-trapecio-trapezoide".

La artrodesis de la articulación mediocarpiana anula el movimiento de lanzamiento de dardos descrito por García-Elías y cols ${ }^{12}$ y aumenta la fuerza de carga en la fosa semilunar del radio en un $22 \%$, descargando la presión sobre la fosa escafoidea sin alterar la distribución de cargas sobre la articulación radiocubital distal'3. De esta forma, si mantenemos un eje radial estable e indoloro podemos conservar hasta un máximo del $80 \%$ de las fuerzas que se transmiten en la articulación radiocarpiana.

\section{TÉCNICA QUIRÚRGICA}

Se realiza un abordaje dorsal longitudinal medio centrado sobre la articulación radiocarpiana. Se procede a disección del tejido subcutáneo hasta la exposición de todo el retináculo extensor. El retináculo se incide entre el tercer y cuarto compartimento. Posteriormente el retináculo extensor se levanta del tubérculo de Lister lo que permite sacar el tendón del músculo Extensor Pollicis Longus del tercer compartimento y separarlo radialmente. Se abre entonces el cuarto compartimento sacando los tendones del Extensor Digitorum Comunis que se rechazan cubitalmente. Si bien es un gesto discutido, La neurectomía del nervio interóseo posterior refuerza el efecto analgésico al realizar una denervación parcial de la muñeca.

En este momento asociamos una neurectomía del nervio interóseo posterior en la zona más proximal, dejando el muñón del nervio lo más profundo posible. La capsulotomía dorsal se puede realizar respetando los ligamentos radiopiramidal dorsal e intercarpiano según describieron Berger y Bishop ${ }^{14}$. Nosotros preferimos para esta artrodesis una incisión de la cápsula en forma de " $Z$ " con un corte longitudinal medio centrado sobre el semilunar @ Figura 3. Se hace una incisión hacia radial desde el vértice proximal desinsertando la 


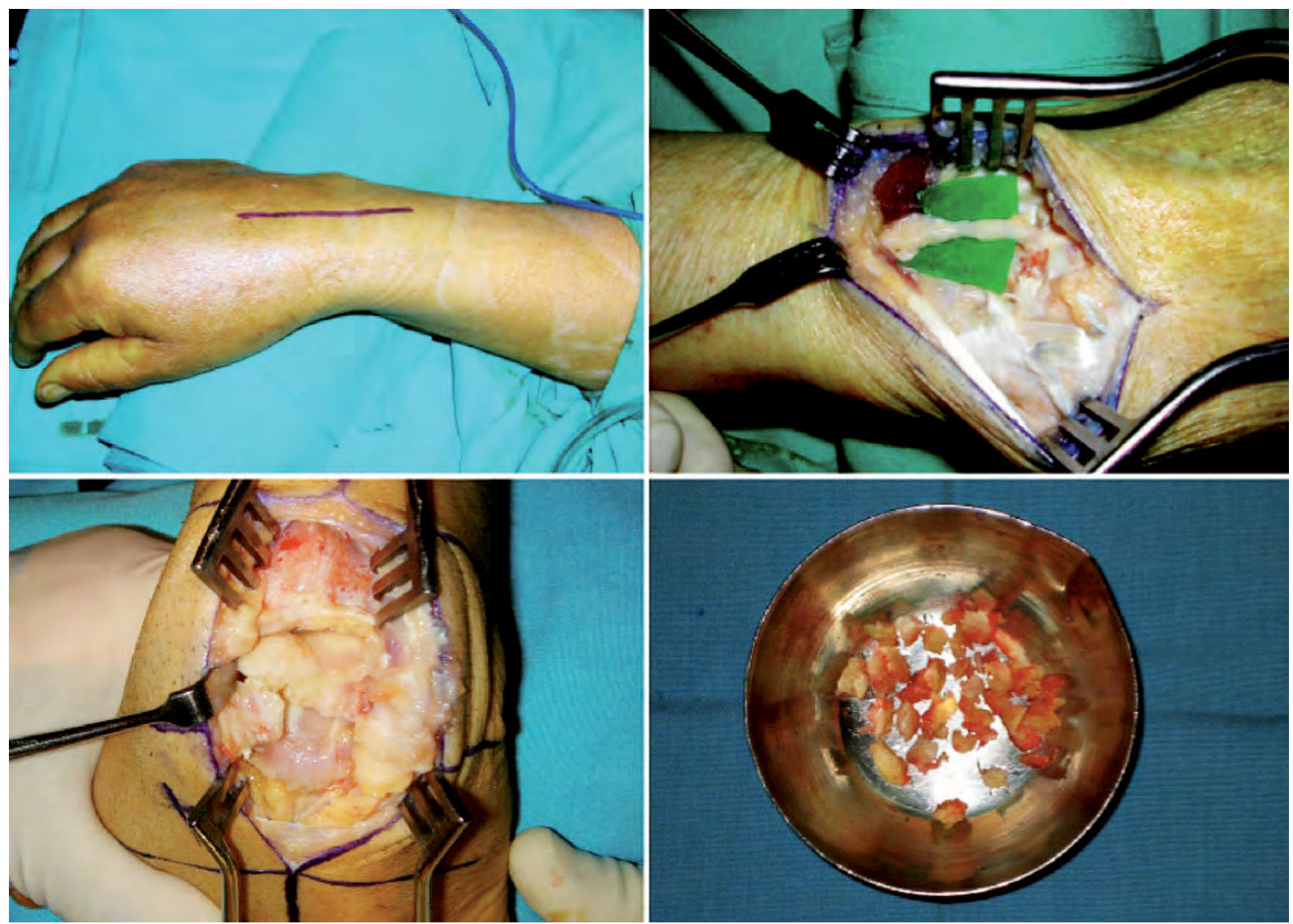

Figura 3. Técnica quirúrgica de la artrodesis de las cuatro esquinas. Incisión longitudinal dorsal sobre el III-IV compatimento extensor y denervación de la rama distal del nervio interóseo posterior. Se realiza una capsulotomía dorsal (preferiblemente en ventana), se procede a la extracción del escafoides carpiano, que una vez desmenuzado permite obtener una cantidad suficiente de injerto que se interpondrá entre las carillas articulares cureteadas.

cápsula del radio, que respeten de esta forma las fibras más cubitales del ligamento radiopiramidal dorsal y se completa con una incisión hacia cubital desde el vértice distal. De esta forma se levantan dos solapas triangulares de cápsula que exponen ampliamente los cuatro huesos que se pretende artrodesar y el escafoides.

Una vez expuestos los huesos carpianos se examinan las superficies articulares para confirmar la extensión de las lesiones. Se realiza la extirpación completa del escafoides con una pinza gubia teniendo cuidado de no dañar los ligamentos extrínsecos. No es necesario asociar estiloidectomía radial. En los casos donde no exista artrosis radioescafoidea no es necesario la extirpación del escafoides como sucede en la artrosis o inestabilidad mediocarpiana. Tampoco es necesaria la extirpación completa de este hueso en el colapso del carpo secundario a una pseudoartrosis de escafoides (muñeca SNAC) pudiendo dejar el polo proximal que conserva su superficie articular indemne.
Se realiza también extirpación con gubia del cartílago articular y hueso subcondral de las áreas de contacto entre los huesos grande, semilunar, piramidal y ganchoso teniendo cuidado en la articulación luno-piramidal de no extirpar la parte más proximal para no dañar la superficie que se articula con el radio. A veces es necesario usar una fresa de alta velocidad o una sierra de pequeño tamaño cuando existe gran esclerosis subcondral. Se fijan temporalmente los cuatro huesos del carpo con agujas de Kirschner teniendo la precaución de colocar el semilunar en posición neutra con respecto al radio ya que la tendencia es a colocarse en dorsiflexión @ Figura 4. Para ello puede ser útil colocar una aguja de Kirchsner en el semilunar para manejarlo como un "joystick". No obstante, se puede variar la posición del semilunar para influir en la movilidad postoperatoria. De Carli y cols. ${ }^{15}$ en un estudio en cadáver obtuvieron una menor movilidad pasiva total en el eje de flexo-extensión cuando el semilunar no estaba colocado en neutro. Sin embargo, se obtuvo 

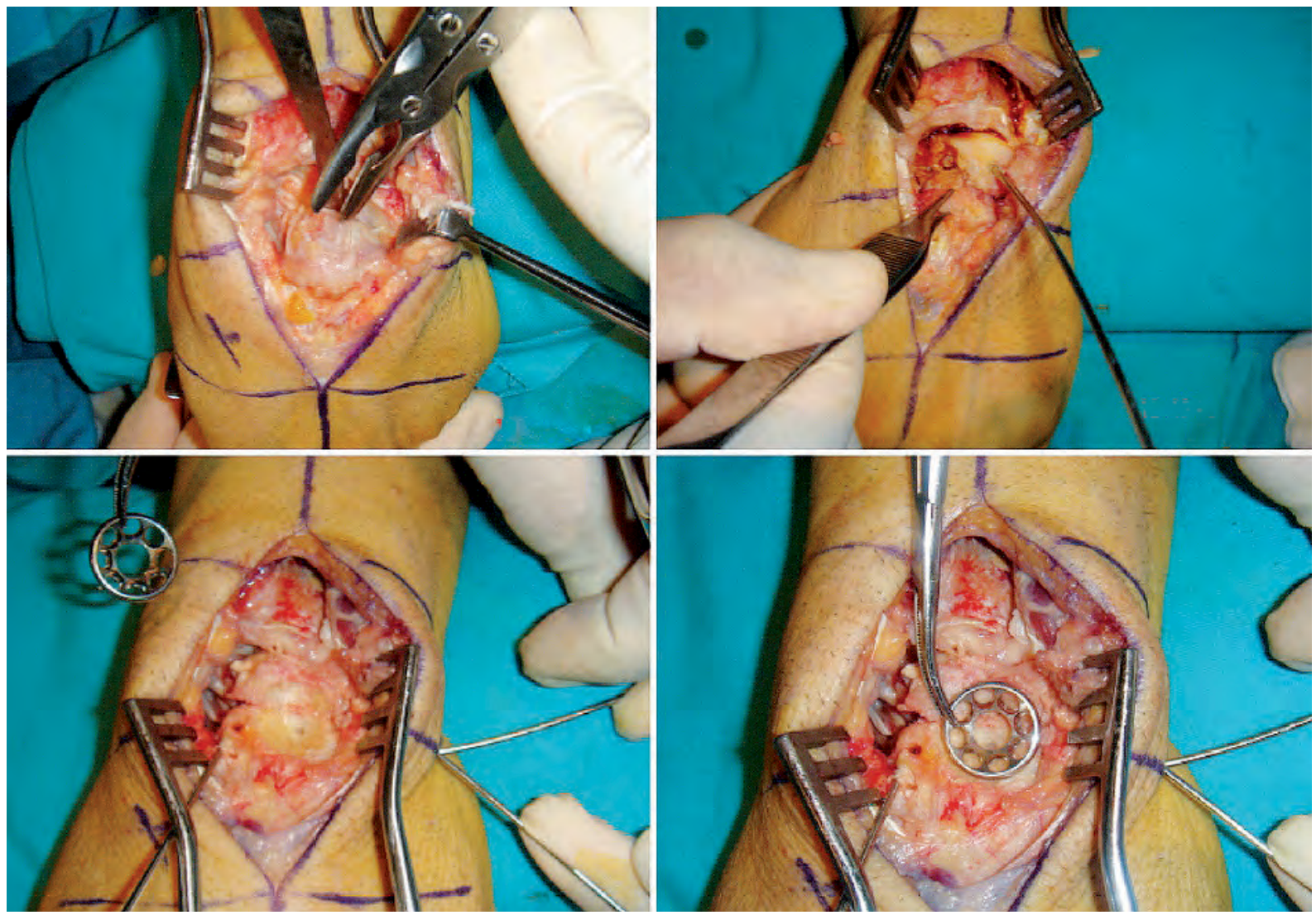

Figura 4. En la artrodesis de cuatro esquinas es importante la resección de la superficie articular de hueso grande, semilunar y piramidal (respetando el ligamento lunopiramidal) así como la corrección de la posición del semilunar. Una vez fijado temporalmente con agujas la artrodesis, se estabiliza con un sistema de fijación estable. En este caso se realizó mediante la placa dorsal circular Spider® (KMI, San Diego).

un incremento de $7^{\circ}$ de la flexión ( $42^{\circ}$ de extensión) cuando se colocaba el semilunar en discreta extensión y $7^{\circ}$ de extensión ( $35^{\circ}$ de flexión) cuando lo hacía en leve flexión respecto al radio. Esto es útil si se pretende obtener un mayor arco de flexión o extensión, en función de las demandas del paciente.

Se comprueba por radioscopia la correcta alineación de los cuatro huesos que se quieren artrodesar y nos fijamos especialmente en la proyección lateral en la posición radio-semilunar-hueso grande en relación $0^{\circ}$. En este momento se realiza la fijación de los huesos del carpo, que puede realizarse con agujas de Kirschner, tornillos canulados, grapas o placas dorsales. Para los casos en los que se decide realizar esta técnica de forma abierta, la fijación con placa circular dorsal atornillada es nuestra técnica de elección. Se obtiene el injerto de escafoides y se introduce entre las superficies articulares, en la unión de la fusión de las cuatro esquinas y en el fondo del defecto raspado. En los casos en que el escafoides se encuentre muy escleroso (lo cual es muy frecuente) o cuando no se vaya a extirpar este hueso, se introduce injerto esponjoso de cresta ilíaca.

Se fresa la cavidad con el instrumental propio de cada implante y se coloca la placa circular que se fija con tornillos, al menos uno a cada uno de los cuatro huesos del carpo. La colocación de la placa es esencial y el borde proximal de la placa debe colocarse en la mitad la superficie dorsal del semilunar y no debe sobrepasar esta línea hacia proximal, para evitar el impacto de la placa con el dorso del radio (1) Figura 5. A la hora de realizar el fresado, no es necesario que sea completo en profundidad y basta con hacerlo hasta el 50\% de la superficie (2) Figura 6. Es muy importante rellenar de injerto todas las superficies a artrodesar y que el primer tornillo que se coloque sea el del semilunar, para evitar que la placa se bascule y pueda originar un choque con el radio. Se debe poner especial atención en el brocado y en la medición del tornillo destinado a fijar el hueso piramidal para evitar dañar la articulación piso-piramidal o que el tornillo sea largo y la punta interfiera en la articulación. 

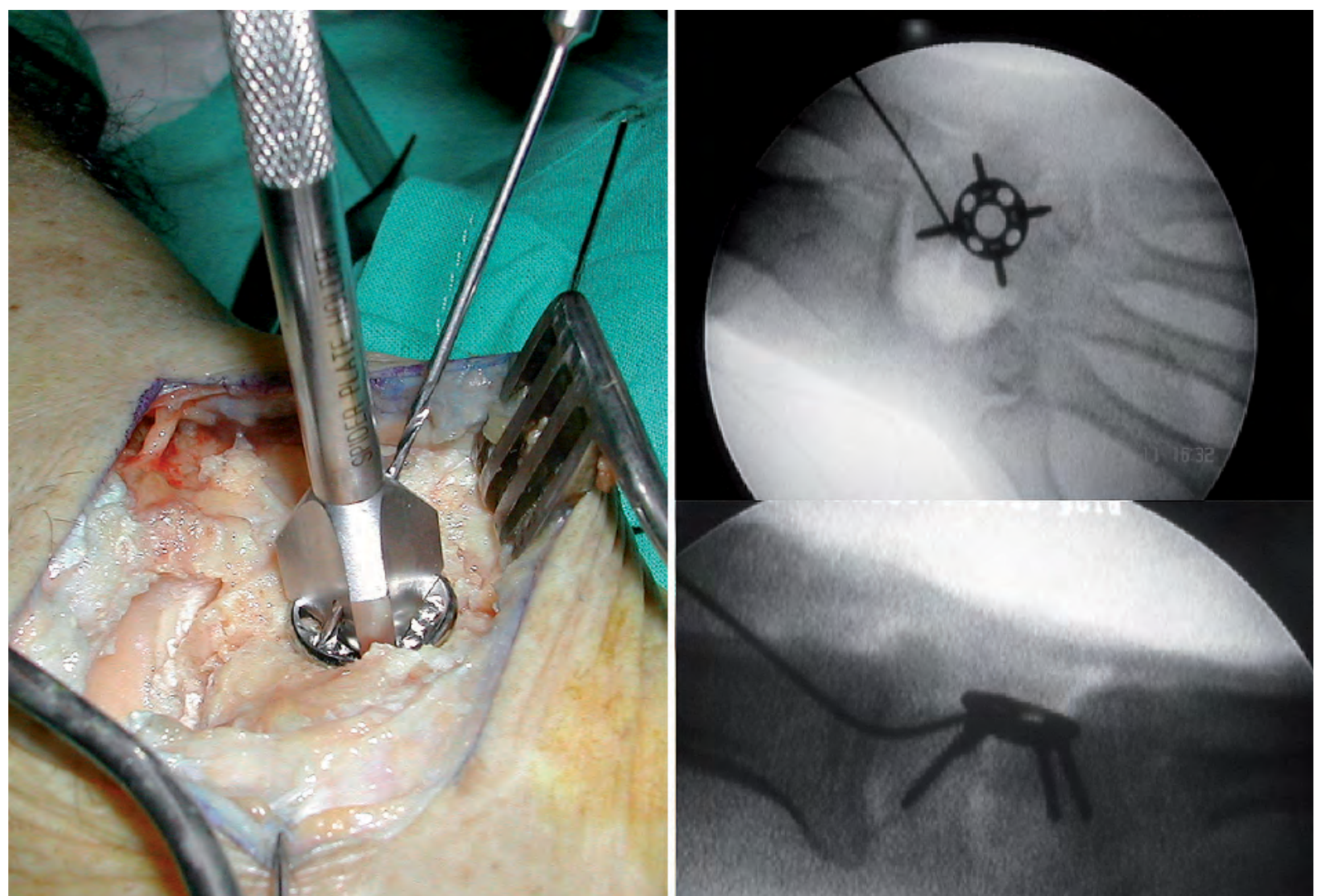

Figura. 5. Artrodesis de cuatro esquinas con placa circular dorsal. Es importante colocar primero el primer tornillo más proximal en semilunar. Se deben fijar al menos con un tornillo cada uno de los cuatro huesos artrodesados.

Se realiza control fluoroscópico para comprobar la posición de la placa y los tornillos, así como pruebas de movilidad para comprobar la estabilidad de la placa y que el borde de la placa proximal o las cabezas de los tornillos no choquen con el labio dorsal del radio en la dorsiflexión. Se cierra la cápsula con puntos sueltos de sutura absorbible y se libera la isquemia para realizar hemostasia y lavado abundante. Se coloca un drenaje en el tejido subcutáneo y se cierra la piel con puntos sueltos de nylon. Realizamos un vendaje generoso e inmovilizamos la muñeca con una férula de yeso en ligera dorsiflexión y desviación cubital de la muñeca. La inmovilización se mantiene durante 3 semanas para que de tiempo a la cicatrización de las partes blandas y se inicie la consolidación y se comienza con la movilización progresiva de la muñeca.

\section{RESULTADOS}

Los resultados obtenidos son variables en función de las series consultadas. La consolidación completa se obtiene a las 10-12 semanas y el dolor disminuye hasta los 2-4 puntos de la escala analógica visual con un arco de movilidad medio final entre $62-84^{\circ}$ de arco de flexo-extensión - Figura 7. Respecto a la función global de la mano se obtienen resultados aceptables, con una fuerza respecto a la muñeca contralateral de $56-78 \%$ y una puntuación final del cuestionario DASH entre 22-45 puntos $4,16,17$.

A pesar de estos buenos resultados funcionales, las complicaciones asociadas a este procedimiento son elevadas. En un estudio retrospectivo de 17 casos tratados con placa dorsal circular, Shindle y cols. ${ }^{18}$ presentan complicaciones en el 55\% de los casos, siendo las más frecuentes la ausencia de consolidación (25\%), el pinzamiento dorsal del radio $(25 \%)$ y la rotura del implante (13\%). Aparte de estas complicaciones, está descrito un $3 \%$ de dolor complejo regional y el $2 \%$ se reconvierten a una fusión total de la muñeca ${ }^{19}$.

El "talón de Aquiles" de esta técnica es la ausencia de consolidación. Algunos trabajos reportan una elevada incidencia de pseudoartrosis en las artrodesis realizadas con placa circular, que contrasta con los buenos 


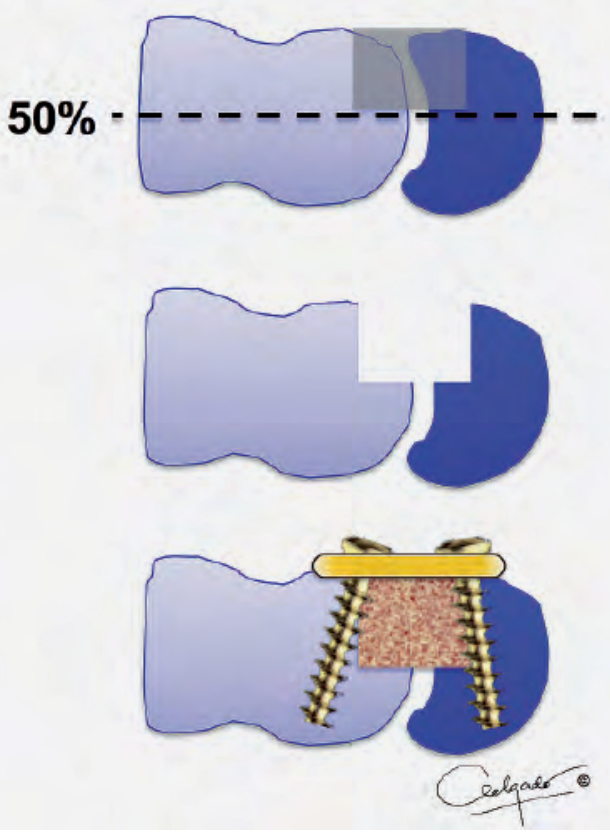

Figura 6. Importante corregir la deformidad dorsal del semilunar y cruentar al menos el $50 \%$ de la superficie dorsal articular. resultados observados en otros trabajos y en nuestra experiencia (-) Tabla II. Esta divergencia en los resultados se puede justificar por la técnica quirúrgica. El mayor porcentaje de complicaciones son atribuidas a la fijación con placas circulares, si bien se trata en general de series con un número escaso de pacientes $^{16-22}$. Vance y cols. ${ }^{23}$ compararon los resultados obtenidos con la artrodesis de cuatro esquinas realizadas con placa circular $(n=27)$ respecto a la fijación con agujas o tornillos $(n=3 \mid$ ). Si bien los resultados fueron similares, obtuvieron peores resultados con la placa circular respecto a ausencia de consolidación (26\% frente a 3\%), puntuación DASH (27 respecto a 8) y grado de satisfacción (60\% contra 100\%).

Es importante realizar un cruentado minucioso de las superficies articulares de los huesos a artrodesar y rellenar bien de injerto los espacios. Como el escafoides con frecuencia está escleroso, proporciona un injerto de mala calidad por lo que en estos casos o cuando se preserva este hueso se recomienda el injerto esponjoso de cresta ilíaca o del radio distal.
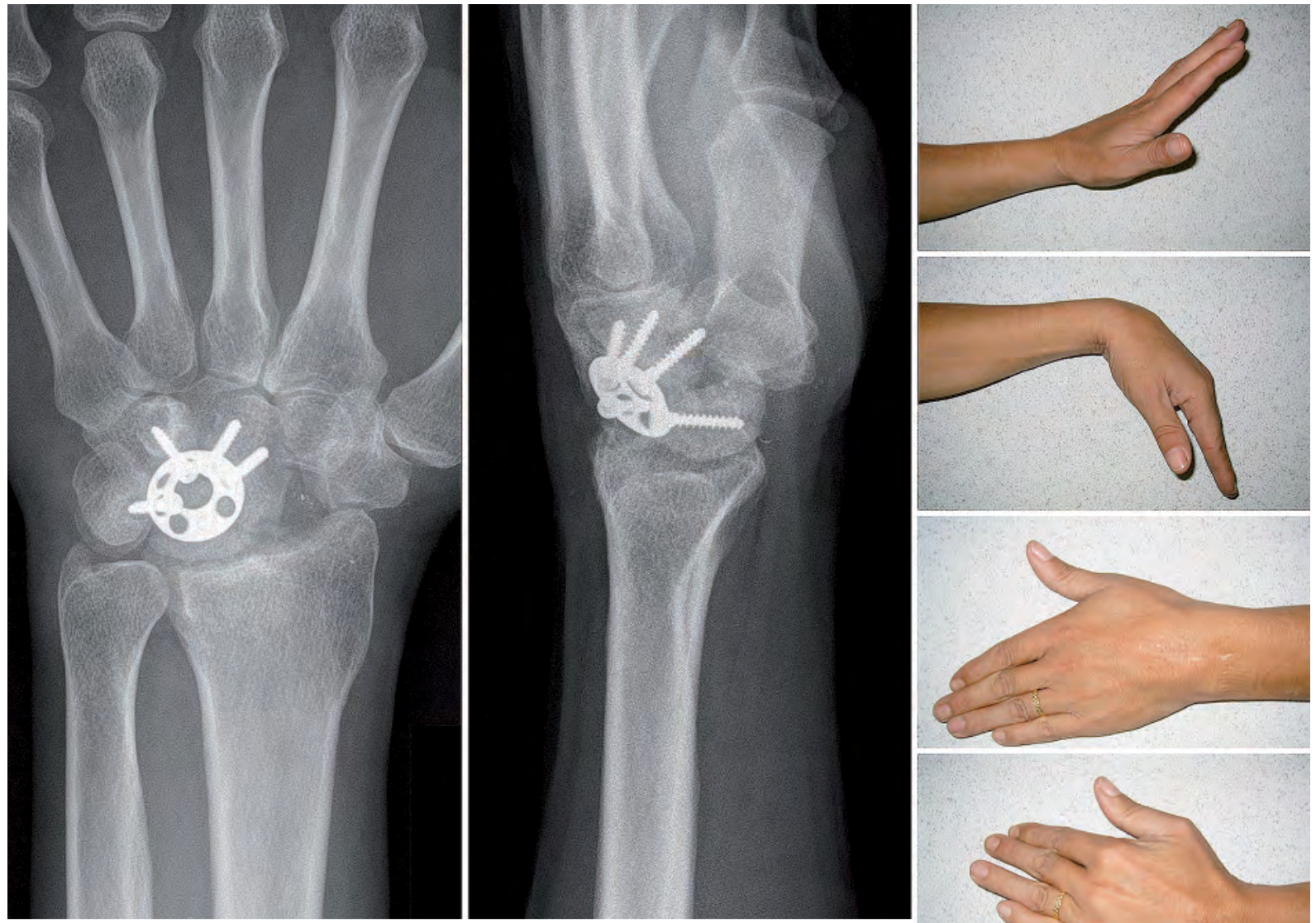

Figura. 7. Artrodesis de cuatro esquinas y fijación con placa circular dorsal atornillada. Aspecto radiológico y funcional a los 4 años.

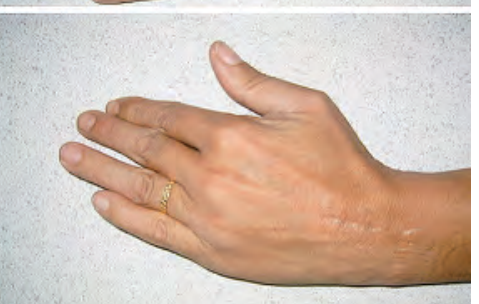




\section{TABLA II - INCIDENCIA DE AUSENCIA DE CONSOLIDACIÓN EN ARTRODESIS DE CUATRO ESQUINAS.}

\begin{tabular}{|c|c|c|}
\hline AUTOR, AÑO & $\mathbf{N}^{\circ}$ PACIENTES & \% NO UNIÓN \\
\hline Watson y cols., $1984^{\prime}$ & 250 & $3 \%$ \\
\hline Ashmead y cols., $1994^{4}$ & 100 & $3 \%$ \\
\hline Krakauer y cols., 199417 & 23 & $6,7 \%$ \\
\hline Cohen y Cozin, 200I ${ }^{20}$ & 19 & $5 \%$ \\
\hline Kendall y cols., $2005^{22}$ & 8 & $62 \%$ \\
\hline Skie y cols., $2007{ }^{13}$ & 37 & $22 \%$ \\
\hline Shindle et al, $2007^{18}$ & 16 & $25 \%$ \\
\hline Gaston y cols., $2009^{21}$ & 18 & $11 \%$ \\
\hline
\end{tabular}

Por otro lado es recomendable inmovilizar la muñeca por 3 semanas, plazo suficiente para que comience la consolidación y comenzar con una rehabilitación progresiva dirigida.

La serie más larga y con más seguimiento es la de Neubrech y cols $^{24}$. En un estudio retrospectivo de 594 artrodesis mediocarpianas en 572 pacientes con un seguimiento mínimo de 10 años (medio de 13,6 años), el $6.7 \%$ de los casos se reconvirtieron a artrodesis total de muñeca, en su gran mayoría al segundo año. En el mismo trabajo, se realizó un estudio clínico transversal de 56 casos a los 14,7 años de seguimiento medio, en los que se observaron un $66 \%$ de cambios degenerativos en la fosa semilunar del radio y un $18,3 \%$ de desviación lunar, a pesar de los buenos resultados clínicos.

En nuestra experiencia, en 36 casos (de 32 años de edad media) tratados de forma consecutiva con placa circular y seguimiento prospectivo medio de 24 meses, hemos obtenido una tasa de consolidación del $95 \%$, $61^{\circ}$ de flexoextensión, 69 puntos en la escala de la Clínica Mayo, 1.92 de escala visual analógica del dolor, $57 \%$ de fuerza y una incorporación a su mismo puesto de trabajo en el $88 \%$ de los casos a las 17 semanas de media 25,26 .

No hay respuesta clara cuando se compara la artrodesis de cuatro esquinas con otras técnicas de salvamento. Respecto a la carpectomía proximal, no se aprecian diferencias significativas respecto a fuerza, dolor y resultado del cuestionario DASH ${ }^{20,27,28}$. Si se compara con la artrodesis capitolunar, no se objetivan diferencias respecto a arco de movilidad, especialmente las desviaciones laterales y pronosupinación 21,29. Esto se atribuye a la conservación del piramidal, que preserva la propiocepción de los ligamentos radiopiramidales ${ }^{30}$. Recientemente se están presentando los resultados de la artrodesis bicolumnar (fijación combinada de capitolunar y pirámido-ganchoso con tornillos independientes), que ofrece resultados funcionales similares a los 2 años a la A4E, pero simplificando la técnica ${ }^{3 !}$.

Sin embargo, estos trabajos aún no explican porqué los pacientes con artrodesis parcial con o sin preservación del piramidal presentan un dolor continuo lacerante en el borde cubital tras la cirugía que persiste a corto-medio plazo.

\section{ARTRODESIS PARCIALARTROSCÓPICA}

La mayoría de las fusiones parciales tienen mayor arco de movilidad en los estudios biomecánicos o durante el acto quirúrgico que el que se obtiene finalmente en el paciente en la práctica habitual. Esto se relaciona con el proceso cicatricial del abordaje dorsal y una menor respuesta elástica compensatoria de las articulaciones adyacentes. 


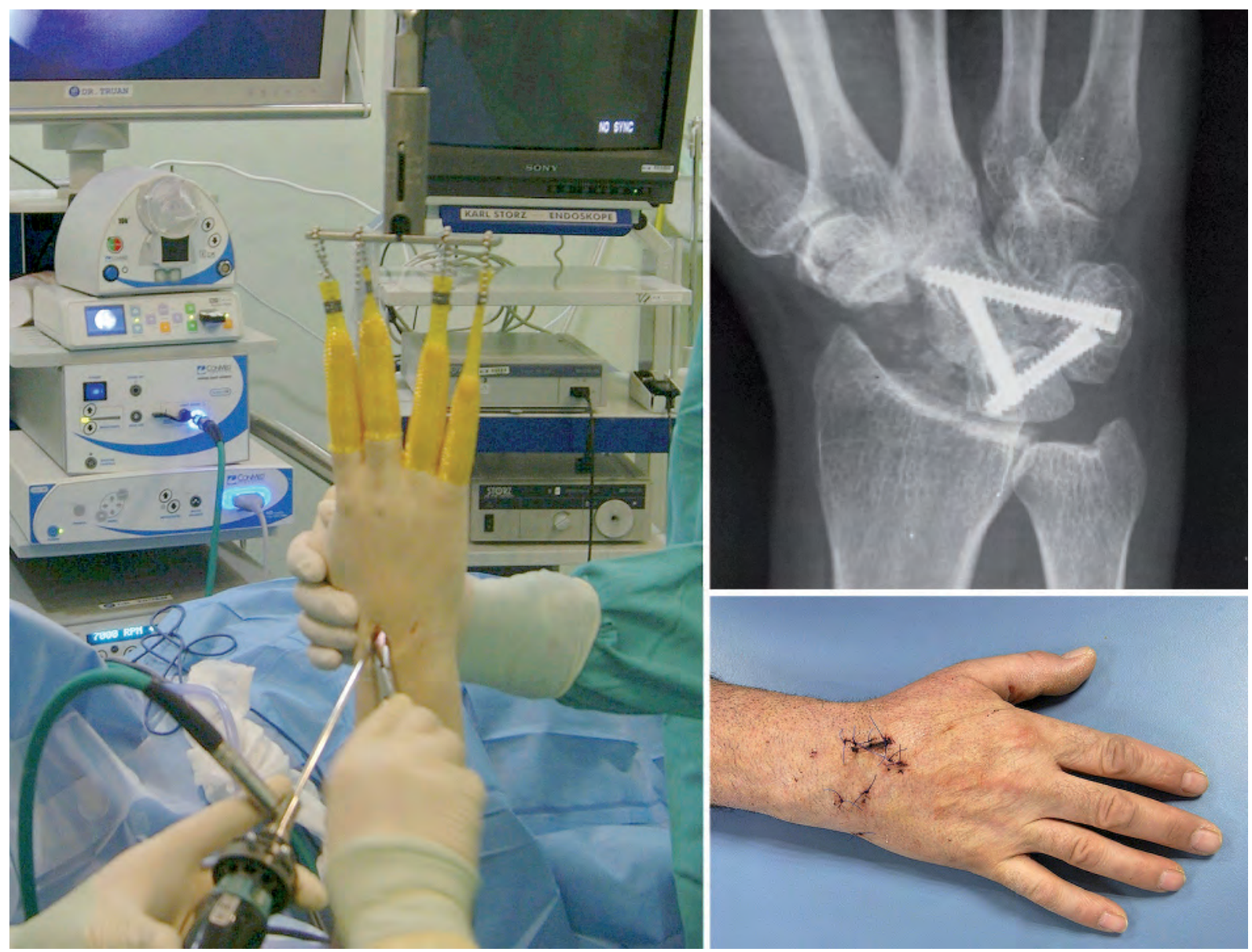

Figura 8. Artrodesis de cuatro esquinas asistida por artroscopia y fijación con tornillos. A la derecha se puede apreciar el resultado radiológico y aspecto de la mano a los 10 días de la intervención, con escasos signos inflamatorios y edema.

Por otro lado, los trabajos de Hagert y cols $^{30,32,33}$ muestran que la lesión del nervio interóseo posterior (que ocurre de forma habitual e intencionada con el abordaje dorsal de la cápsula de Berger y Bishop ${ }^{14}$ ) influye de forma negativa en la propiocepción del ligamento escafolunar, lo que puede condicionar una pérdida de movilidad adaptativa de las articulaciones vecinas, aunque este es un tema que aún se encuentra en discusión y debate ${ }^{34}$.

La artroscopia supone un avance en este aspecto, al realizar un menor daño de las partes blandas y respetar la propiocepción de la muñeca, permite evitar estos riesgos de entrada y un menor tiempo de recuperación. La primera artrodesis mediocarpiana asistida por artroscopia y fijación percutánea fue descrita en 2003 por Slade y Bomback ${ }^{35}$. Con posterioridad, $\mathrm{Ho}^{36,37}$ ha presentado diversas artrodesis asistidas por artroscopia, realizadas bajo anestesia general, sin isquemia e infiltración de los portales con adrenalina, con buenos resultados, pero largos tiempos quirúrgicos (casi 4 horas). Los trabajos de Del Piñal y $\operatorname{cols}^{38,39}$ son los que realmente han impulsado esta técnica gracias a la publicación de detalles técnicos, nuevos portales y el concepto de la artroscopia "seca", que han contribuido a disminuir las complicaciones y los tiempos quirúrgicos de las primeras descripciones.

Los resultados a largo plazo son aún una incognita, pero sus resultados intermedios hacen para nosotros que sea una técnica de elección y se obtiene una recuperación más rápida en nuestra experiencia (d) Figura 8.

Del Piñal y $\operatorname{cols}^{39}$ en 4 pacientes con muñecas SLAC/ SNAC III en los que realizó una fusión de cuatro esquinas con tornillos percutáneos, a los 14 meses de seguimiento medio obtuvo excelentes resultados, presentando un arco de flexo-extensión medio de 73, $43 \mathrm{~kg}$ de fuerza, disminución de dolor hasta 1//0 de escala 
analógico-visual, un 100\% de consolidación y sin complicaciones derivadas del procedimiento.

\section{CONCLUSIONES}

La artrodesis de las cuatro esquinas está indicada en los casos sintomáticos de muñeca SLAC o SNAC grados II y III, o en aquellos casos que precisen una artrodesis parcial y que está preservada la articulación entre radio y semilunar. La utilización de sistemas de fijación estable permite una movilización y rehabilitación más precoz pero hay que hacer un cruentado minucioso de todas las superficies articulares y un relleno de injerto con fragmentos de hueso esponjoso.

Las técnicas artroscópicas son procedimientos muy exigentes que requieren una gran experiencia en el manejo de la técnica, pero minimizan los problemas de partes blandas y propiocepción, con lo que se consigue una recuperación más rápida y con menos complicaciones, por lo que probablemente sea la técnica de elección en el futuro.

\section{CONFLICTOS DE INTERESES}

Los autores declaran no tener conflictos de intereses.

\section{BIBLIOGRAFÍA}

I. Watson HK, Ballet FL. The SLAC wrist: scapholunate advanced collapse pattern of degenerative arthritis. J Hand Surg. 1984;9:358-365.

2. Kobza PE, Budof JE, Yeh ML, Luo ZP. Management of the scaphoid during four-corner fusion. A cadaveric study.J Hand Surg Am. 2003;28:904-9.

3. García-López A, Delgado PJ. Artrodesis parciales: fusión de las cuatro esquinas. Rev Ap Locomot. 2007;5(supl II):97- 104.

4. Ashmead D, Watson HK, Damon C, Herver S, and Paly W. Scapholunate advanced collapse wrist salvage. J Hand Surg Am. 1994; 19:74I-750.

5. García López A., Pérez-Ubeda M.J., Marco F., Molina. M and López-Duran L. A modified technique of four-bone fusion for advanced carpal collapse (SLAC/SNAC wrist). J Hand Surg Br. 200 I;26:352354.
6. Brumfield RH, Champoux JA. A Biomechanical study of normal functional wrist motion. Clin Orthop. 1984; 187:23-5.

7. Palmer AK, Werner FW, Murphy D, Glisson R. Functional wrist motion: a biomechanical study. J Hand Surg Am. 1985; 10:39-46.

8. Nelson DL. The functional range of motion of the wrist. American Society for Surgery of the Hand. 45th Annual Meeting. Toronto, 1990.

9. Ryu J, Cooney WP, Askew IJ, An KN, Chao EYS. Functional ranges of motion of the wrist joint. J Hand Surg Am. 199|; 6:409-19.

10. García-Elías M, Cooney W, An K, Linscheid RL, Chao EY.Wrist kinematics after limited intercarpalarthrodesis, J Hand Surg Am. 1989; | 4:79 |-9.

I I. Iwasaki N, Minami A, Miyazawa T, Kaneda K. Force distribution through the wrist joint in patients with different stages of Kienböck disease: Using computed tomography osteoabsorptiometry. J Hand Surg Am. 2000;28:870-876.

12. García-Elías, M. Kinetic análisis of carpal stability during grip. Hand Clin. 1997; I 3: I 5 I-8.

13. Skie M, Grothaus M, Ciocanel D, Goel V. Scaphoid excision with four-corner fusion: a biomechanical study. Hand (NY). 2007;32: 1256-62.

14. Berger RA, Bishop AT, Bettinger PC. New dorsal capsulotomy for the surgical exposure of the wrist. Ann Plast Surg. 1995;35:54-9.

15. De Carli P, Donndorff AG, Alfie V, Boretto JG, Lopez-Ovenza JM, Gallucci GL. Four-corner arthrodesis: influence of the position of the lunate on postoperative wrist motion. J Hand Surg Am. 2007;32:1 356-62.

16. De Francisco B, García-López A, López Y, Otero J, López-Duran L. Artrodesis mediocarpiana con placa Spider®. Rev Ortop Traumatol. 2007;2:6974.

17. Krakauer JK, Bishop AT, Coonet WP. Surgical treatment of scapholunate advanced collapse. J Hand Surg Am. 1994; 19:751-9.

18. Shindle MK, Burton KJ, Weiland AJ, Domb BG, Wolfe SW. Complications of circular plate fixation for four-corner arthrodesis. J Hand Surg Eur Vol. 2007;32:50-3.

19. Shin AY. Four-corner arthrodesis. J Am Soc Surg Hand. 200 I; I:93- I I I.

20. Cohen MS, Cozin SH. Degenerative arthritis of the wrist: proximal row carpectomy versus scaphoid excision and four-corner arthrodesis. J Hand Surg Am 200 I;26:94-104. 
21. Gaston RG, Greenberg JA, Baltera RM, Mih A, Hastings $\mathrm{H}$. Clinical outcomes of scaphoid and triquetral excision with capitolunate arthrodesis versus scaphoid excision and four-corner arthrodesis. J Hand Surg Am 2009;34A: I 407- 12

22. Kendall CB, Brown TR, Millon SJ, Sanders JL, Tanner SL. Results of four-corner arthrodesis using dorsal circular plate fixation. J Hand Surg Am. 2005;30:903-7.

23. Vance MC, Hernández JD, Didonna ML, Stern PJ Complications and outcome of four-corner arthrodesis: circular plate fixation versus traditional techniques. J Hand Surg Am. 2005;30: I 1 22-7.

24. Neubrech F, Mühldorfer-Fodor M, Pillucat T, van Schoonhoven J, Prommersberger KJ. Long-term results after midcarpal arthrodesis. J Wrist Surg. 2012;1:123-128.

25. Delgado PJ, García-López A, De Felipe J, Fuentes A, López-Oliva F. The relationship between 4-corner wrist arthrodesis and heavy-labour workers. J Bone Joint Surg. 2009;9I-B(Supl I):I 54-5.

26. Delgado PJ:Tratamiento de las artropatías degenerativas de la muñeca. En: Manual de cirugía ortopédica y traumatología. SECOT. $2^{a}$ edición. Madrid: Panamericana, 20 I0; p. 979-85.

27. Mulford JS, Ceulemans LJ, Nam D, Axelrod TS. Proximal row carpectomy vs four corner fusion for scapholunate (SLAC) or scaphoid non-union advanced collapse (SNAC) wrists: a systematic review of outcomes. J Hand Surg Eur Vol. 2009;34:256-63.

28. Vanhove W, De Vil J, Van Seymortier P, Boone B, Verdonk R. Proximal row carpectomy versus four-corner arthrodesis as a treatment for SLAC (scapholunate advanced collapse) wrist. J Hand Surg EurVol. 2008;33: I 18-25.

29. Ferreres A, García-Elías M, Plaza R. Long-term results of lunocapitate arthrodesis with scaphoid ex- cision for SLAC and SNAC wrists. J Hand Surg Eur. 2009;34:603-8.

30. Hagert E, García-Elías M, Forsgren S et al. Inmunohistochemical analysis of wrist ligament innervation in relation to their structural composition. J Hand Surg Br. 2007:32:30-6.

31. Draeger RW, Bynum DK, Schaffer A, Patterson JM. Bicolumnar intercarpal arthrodesis: mínimum 2-year follow-up. J Hand Surg Am. 20 I4;39:888-94.

32. Hagert E, Persson JK, Werner M, Ljung BO, Evidence of wrist proprioceptive reflexes elicited after stimulation of the scapholunate interosseous ligament. J Hand Surg Am. 2009;34:642-5I.

33. Hagert E. Proprioception of the wrist joint: a review of current concepts and possible implications on the rehabilitation of the wrist. J Hand Ther. 2010;23:2-1.

34. Dellon AL. Commentary: desensitizing the posterior interosseous nerve alters wrist propioceptive reflexes: it is OK to lose your nerve. J Hand Surg Am. 20 10;35: 67-9.

35. Slade JF, Bomback. Percutaneous capitolute arthrodesis using arthroscopic or limited approach. Atlas Hand Clin. 2003;8: 1 49-62.

36. Ho PC. Arthroscopic partial wrist fusion. Tech Hand Upper Extrem Surg. 2008;242-65.

37. Ho PC. Arthroscopic radiocarpal fusion for posttraumatic radiocarpal arthrosis. E: Del Piñal $F(e d)$. Arthroscopic management of distal radius fractures. Springer-Verlag: Berlin 20 10;p.225-42.

38. Del Piñal, F. Dry artroscopy and its applications. Hand Clin. 201 1;27:334-45.

39. Del Piñal F, Klausmeyer M, Thams C, Moraleda E, Galindo C. Early experience with (dry) arthroscopy 4-corner arthrodesis: from a 4-hour operation to a tourniquet time. J Hand Surg Am. 2012:37:2389-99. 